\title{
Environment, morphology, and stellar populations of bulgeless low surface-brightness galaxies
}

\author{
X. Shao ${ }^{1,2,3}$, K. Disseau ${ }^{2}$, Y. B. Yang ${ }^{2}$, F. Hammer ${ }^{2}$, M. Puech ${ }^{2}$, M. Rodrigues ${ }^{2}$, Y. C. Liang ${ }^{1}$, and L. C. Deng ${ }^{1}$ \\ ${ }^{1}$ Key Laboratory of Optical Astronomy, National Astronomical Observatories, Chinese Academy of Sciences, 20A Datun Road, \\ Chaoyang District, 100012 Beijing, PR China \\ e-mail: xshao@bao.ac.cn \\ 2 Laboratoire GEPI, Observatoire de Paris, CNRS-UMR 8111, Univ. Paris Diderot, Sorbonne Paris Cité, 5 place Jules Janssen, \\ 92195 Meudon, France \\ ${ }^{3}$ University of the Chinese Academy of Sciences, 100049 Beijing, PR China
}

Received 3 February 2015 / Accepted 22 April 2015

\begin{abstract}
Based on the Sloan Digital Sky Survey DR 7, we investigate the environment, morphology, and stellar population of bulgeless low surface-brightness (LSB) galaxies in a volume-limited sample with redshift ranging from 0.024 to 0.04 and $M_{r} \leq-18.8$. The local density parameter $\Sigma_{5}$ is used to trace their environments. We find that, for bulgeless galaxies, the surface brightness does not depend on the environment. The stellar populations are compared for bulgeless LSB galaxies in different environments and for bulgeless LSB galaxies with different morphologies. The stellar populations of LSB galaxies in low-density regions are similar to those of LSB galaxies in high-density regions. Irregular LSB galaxies have more young stars and are more metal-poor than regular LSB galaxies. These results suggest that the evolution of LSB galaxies may be driven by their dynamics, including mergers rather than by their large-scale environment.
\end{abstract}

Key words. galaxies: evolution - galaxies: formation - galaxies: kinematics and dynamics - galaxies: spiral - galaxies: star formation

\section{Introduction}

Low surface brightness (LSB) galaxies usually refer to galaxies that possess an exponential disk with a face-on central surface brightness fainter than the ambient night sky. This type of galaxy has been widely discussed after Freeman (1970) found that 28 of 36 disk galaxies have central surface brightness in the range $\mu_{0}(B)=21.65 \pm 0.30 \mathrm{mag} \operatorname{arcsec}^{-2}$. Disney (1976) showed that this result is biased by a strong selection effect, which hinders the discovery of LSB galaxies. Thanks to improvements in telescopes and instruments, much has been done to understand this kind of galaxy (Schombert \& Bothun 1988; Caldwell \& Bothun 1987; Schombert et al. 1992). McGaugh et al. (1995b) found that the morphologies of LSB galaxies span a wide range from dwarfs and irregulars to disk galaxies. Regardless of their morphology and size, all LSB galaxies have low star formation rates, low metallicities, and extended HI gas disks (Impey \& Bothun 1997).

There is no conventional definition for LSB galaxies yet (Impey \& Bothun 1997), although they have been intensively studied. The most common thresholds of $\mu_{0}(B)$ for LSB galaxies found in the literature are between 22 and $23 \mathrm{mag} \mathrm{arcsec}^{-2}$ (McGaugh et al. 1995b; de Blok et al. 1995; Rosenbaum \& Bomans 2004; Galaz et al. 2011). Since the definition of LSB galaxies only depends on the central surface brightness, more work needs to be done to understand whether LSB galaxies trace the formation and evolution of galaxies in a more representative manner than do high surface brightness (HSB) galaxies or if they just follow the trends of HSB galaxies. This is crucial for understanding the formation and evolution of galaxies.

The nature of the local and large-scale environment in which LSB galaxies are embedded has been analyzed by Bothun et al. (1993) and Mo et al. (1994). They found that LSB galaxies are located in more isolate environments than are HSB galaxies. The deficiency of nearby companions for LSB galaxies was discovered by Zaritsky \& Lorrimer (1993). Recently, based on the large amount of data released by the SDSS, the influence of environment on the evolution of LSB galaxies has been further revealed. Using the Early Data Release of the SDSS, Rosenbaum \& Bomans (2004) show significant differences in local density between LSB and HSB galaxies on scales from 2 to $5 \mathrm{Mpc}$. In their subsequent analysis, Rosenbaum et al. (2009) compared LSB galaxies at $0.01<z \leq 0.055$ and at $0.055<z \leq 0.1$. The results suggest that LSB galaxies formed in a low-density region of the initial universe and then drifted to the outer parts of the filaments and walls of large-scale structures.

In addition to this issue, the origin of the LSB phenomenon is still unknown. Could LSB galaxies result from a similar formation scenario to HSB galaxies? Considerable progress has been made in understanding the formation of HSB disk galaxies. In examining the progenitors of the latter, Hammer et al. (2005) have suggested that their disks have been rebuilt after a past collision of gas-rich mergers. This has been supported by the strong evolution of HSB disk morphologies revealed by Delgado-Serrano et al. (2010), showing that half of the disk progenitors were morphologically peculiar 6 Gyr ago. In fact, the 
combination of morphology and kinematics (Neichel et al. 2008; Hammer et al. 2009) convincingly demonstrate that a significant fraction of present-day disks were formed after being reprocessed through a gas-rich major merger, in agreement with expectations from $\Lambda$ CDM (Puech et al. 2012). This has been discussed by Kormendy et al. (2010), who claim that most local disks do not have a classical bulge, implying that they could not be related to mergers. However, the question needs further clarification because pseudo bulges or even bulgeless galaxies can be formed after very gas-rich mergers (e.g., Hammer et al. 2012; Keselman \& Nusser 2012).

As part of our series of papers (Zhong et al. 2008 and Liang et al. 2010 that analyzed metallicities in LSB galaxies based on a large sample), we would like to analyze the environment, morphology, and stellar populations of bulgeless LSB galaxies. Bulgeless galaxies have lower average surface brightness than galaxies with bulges (Sachdeva 2013). The simulations of Governato et al. (2010) show that strong outflows from star formation can drag dark and luminous matter with them to remove matter with low angular momentum and prevent the formation of a high-density core or bulge. This is supported by Walcher et al. (2005), who found massive star clusters at the cores of bulgeless galaxies. The analysis of central star formation in relation to the environment and dynamics may be helpful for understanding the evolution of bulgeless LSB galaxies.

When analyzing stellar populations using SDSS spectra, the selection of bulgeless LSB galaxies has the advantage that most of the light covered by the SDSS fiber will sample the properties of central disks without contaminations from the bulge light. Several studies have found that LSB galaxies are dominated by bulgeless galaxies. McGaugh et al. (1995b) analyzed the images of 22 LSB galaxies and found that most of them show $B / D<0.1$. O'Neil et al. (1997) analyzed the morphology of 127 LSB galaxies and found that the majority of them $(80 \%)$ were well fit by a single exponential profile. This implies that studying a large sample of bulgeless LSB galaxies may significantly help in general for understanding the properties of LSB galaxies.

Our bulgeless LSB galaxies are selected from the nearby universe. The local densities are calculated to see in what kinds of environment bulgeless LSB galaxies are located. Their stellar populations will be analyzed to see which factor, environment or dynamics, drives the evolution of bulgeless LSB galaxies. This paper is organized as follows. In Sect. 2, we describe the selection of our samples. The relations between surface brightness of bulgeless galaxies and their environment are investigated in Sect. 3. In Sect. 4, we analyze the stellar populations of bulgeless LSB galaxies and see whether their evolution is driven by their environment or by their morphological behavior. Finally, we discuss our results and summarize our conclusions in Sects. 5 and 6, respectively. Throughout the paper, a cosmological model with $H_{0}=70 \mathrm{~km} \mathrm{~s}^{-1} \mathrm{Mpc}^{-1}, \Omega_{\mathrm{M}}=0.3$, and $\Omega_{\Lambda}=0.7$ has been adopted.

\section{The sample}

\subsection{Volume-limited bulgeless sample}

The bulgeless LSB galaxies analyzed in this work are obtained from the main-galaxy sample of SDSS Data Release 7 (York et al. 2000; Stoughton et al. 2002; Strauss et al. 2002; Abazajian et al. 2009). The SDSS is the most ambitious astronomical survey ever undertaken in imaging and spectroscopy, and it targets hundreds of thousands of galaxies. The imaging data, which are

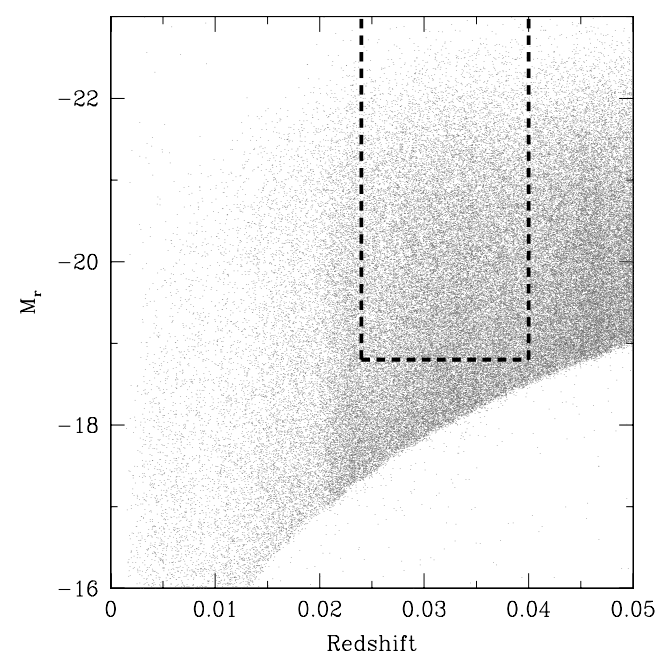

Fig. 1. Location of our volume-limited sample in the SDSS main sample (surrounded by a black dashed line).

obtained in drift scan mode and have covered more than $10^{4} \mathrm{deg}^{2}$ until now, are $95 \%$ complete for point sources at magnitudes of 22.0, 22.2, 22.2, 21.3, and 20.5 in five bands (ugriz). Our samples are selected following the criteria below.

1. We constructed a volume-limited sample to investigate the environments and analyze the stellar populations. A volumelimited sample eliminates the luminosity bias in the SDSS database. The redshift range of this sample is $0.024<z<$ 0.04 , and the corresponding absolute magnitude limit is $M_{r} \leq-18.8$. The lower redshift limit avoids effects from proper motions, which are not negligible in the nearby universe. The upper redshift limit is set to include as many faint galaxies as possible when studying the environment. In Fig. 1, we show the limits of our volume-limited sample (sample $S_{0}$ ).

2. Bulgeless galaxies were selected from this volume-limited sample. Strictly, decomposition is a good method of estimating the portion of bulge light and disk light in one galaxy. Here we use the parameter fracDeV $V_{r}$, which is provided by the SDSS database, to select bulgeless galaxies. However, fracDeV is a qualitative indicator of the existence of a bulge, so it cannot be used to represent the fraction of the bulge light quantitatively. Considering the definition of fracDeV ${ }^{1}$, only for the case of fracDeV $=0$ does it correspond to a pure disk galaxy without a bulge. We therefore chose galaxies with fracDeV $V_{r}=0$ in our volume-limited sample and get the bulgeless galaxies.

3. Low-inclination galaxies are required to avoid the possible bias caused by internal extinction. As pointed out by Unterborn \& Ryden (2008), extinction related to the inclination becomes important when the axis ratio $b / a$ decreases ( $a$ and $b$ are the semimajor and semiminor axis, respectively). But for galaxies with $b / a>0.5$, extinction related to the inclination cannot be distinguished from intrinsic extinction. We therefore limited our sample to $b / a>0.5$ to avoid any bias.

As a result, we selected a bulgeless (fracDeV $\left.V_{r}=0\right)$ and low inclination $(b / a>0.5)$, volume-limited $(0.024<z<$ $\left.0.04, M_{r} \leq-18.8\right)$ sample from SDSS DR7 to investigate the

$1 F_{\text {composite }}=$ fracDeVF $F_{\mathrm{deV}}+(1-$ fracDeV $) F_{\text {exp }}$. 
Table 1. Number of galaxies in the different samples.

\begin{tabular}{ccc}
\hline \hline Sample & Number & Description \\
\hline$S_{0}$ & 31674 & Volume-limited sample \\
$S_{1}$ & 2606 & Bulgeless volume-limited sample \\
$S_{\text {LSB }}$ & 1235 & Bulgeless LSB galaxies \\
$S_{\text {HSB }}$ & 1371 & Bulgeless HSB galaxies \\
\hline
\end{tabular}

environment and stellar populations of LSB galaxies. The number of galaxies selected from the different criteria are listed in Table 1.

\subsection{Low surface brightness sample}

The next step after selecting a volume-limited sample is to calculate the surface brightness and classify the whole sample into LSB galaxies and HSB galaxies. For pure disk galaxies, the profile shape is assumed to be exponential:

$\Sigma(r)=\Sigma_{0} \exp (-r / \alpha)$

where $\Sigma_{0}$ is the central surface brightness of the disk in units of $M_{\odot} \mathrm{pc}^{-2}$, and $\alpha$ is the scale length of the disk. In logarithmic units, the exponential profile is

$\mu(r)=\mu_{0}+1.086(r / \alpha)$

where $\mu_{0}$ is the central surface brightness in mag $\operatorname{arcsec}^{-2}$ (McGaugh et al. 1995a). The total flux is

$F_{\text {tot }}=2 \pi \alpha^{2} \Sigma_{0}$

if the disk is assumed to be infinitely thin. By including the correction of extinction and cosmological dimming effect (Phillipps et al. 1990), we get the general formula to calculate central surface brightness:

$\mu_{0}(m)=m_{\text {disk }}+2.5 \log \left(2 \pi \alpha^{2}\right)+\mathcal{E}(b / a)-10 \log (1+z)$

where $m_{\text {disk }}$ refers to the apparent magnitude of the disk, $\mathcal{E}(b / a)$ denotes extinction related to the inclination, and $z$ is the redshift. Our sample consists of bulgeless galaxies, so the total apparent magnitude of galaxies is denoted as $m_{\text {disk }}$. For galaxies with $b / a>0.5$, extinction related to the inclination cannot be distinguished from the intrinsic extinction (Unterborn \& Ryden 2008), so the extinction correction $\mathcal{E}(b / a)$ is neglected in our sample when calculating the central surface brightness. The $B$-band surface brightness is calculated from magnitudes in $g$ and $r$ bands of the SDSS system by using the conversion provided by Smith et al. (2002).

The widely used definition of LSB galaxies is to select them as $1 \sigma$ fainter than the median value of the Freeman law $(21.65 \pm$ $0.3 \mathrm{mag} \mathrm{arcsec}^{-2}$ ). However, this is not an ideal definition, because Freeman's sample was not complete. McGaugh et al. (1995a; see their Fig. 3) and O'Neil \& Bothun (2000; see their Fig. 2) estimated the distribution of surface brightness in more complete samples. The space densities of galaxies with $\mu_{0}(B)<$ 21.65 mag $\operatorname{arcsec}^{-2}$ become lower when $\mu_{0}(B)$ decreases. For galaxies with $\mu_{0}(B)>21.65 \mathrm{mag} \operatorname{arcsec}^{-2}$, their space densities are almost constant up to $\mu_{0}(B)=25 \mathrm{mag} \mathrm{arcsec}^{-2}$. Using the same definition as de Blok et al. (1995), MorshidiEsslinger et al. (1999), and Rosenbaum \& Bomans (2004), we define LSB galaxies as $\mu_{0}(B)>22.5 \mathrm{mag} \operatorname{arcsec}^{-2}$ and get 1235 LSB galaxies (sample $S_{\text {LSB }}$, see Table 1 ). This definition is $3 \sigma$ fainter than the median value of the Freeman law $\left(21.65 \mathrm{mag} \operatorname{arcsec}^{-2}\right)$. For the rest - i.e., 1371 galaxies with $\mu_{0}(B)<22.5 \mathrm{mag} \operatorname{arcsec}^{-2}$ - we define them as HSB galaxies (sample $S_{\mathrm{HSB}}$ ). But we should note that the HSB galaxies defined that way are not all high surface brightness galaxies, since some LSB galaxies could be part of them if one follows the classical definition of $1 \sigma$ below $21.65 \mathrm{mag} \mathrm{arcsec}^{-2}$. However, this does not affect our results when we analyze the dependence between surface brightness and environment by dividing these bulgeless galaxies into two parts.

\subsection{Selection effects}

Since we selected bulgeless LSB galaxies in a volume-limited sample, the possible selection effects of our sample needs to be checked. We used fracDe $V_{r}=0$ to define bulgeless LSB galaxies, so some bulgeless LSB galaxies with fracDeV $V_{r}>0$ might still have been excluded from our sample. Unfortunately, we have not found a complete sample of LSB galaxies with the same selection criteria as our sample with which we can compare. Disseau et al. (in prep.). selected a representative sample of 150 galaxies from a volume-limited sample with $0.022<z<0.033$ and $M_{r} \leq-18$. Although this sample was not selected in exactly the same way, we used it to roughly estimate the selection bias. To remove the extinction bias, they selected 68 low inclination galaxies with $b / a>0.5$. Using bulge-disk decomposition, they calculated the surface brightness and finally obtained 11 low-inclination LSB galaxies by defining $\mu_{0}(r)>21.7 \mathrm{mag} \mathrm{arcsec}^{-2}$. The fraction of LSB galaxies in their volume-limited sample is $\sim 16.2 \pm 4.9 \%$. In our work, we obtain 1235 bulgeless LSB galaxies in the volume-limited sample of 31674 galaxies (which contains 13731 low-inclination galaxies). The fraction of bulgeless LSB galaxies is $\sim 9.0 \pm 0.2 \%$. Compared with these two samples, the fraction of LSB galaxies only differs by $\sim 1.5 \sigma$, indicating that perhaps we may have lost $\sim 40 \%$ of bulgeless LSB galaxies by using the fracDeV $V_{r}=0$ criterion.

Figure 2 shows the histogram distribution of redshifts for LSB and HSB galaxies in sample $S_{1}$ and their fraction of the whole sample as a function of redshift. In the lefthand panel, one can see that the number in our sample grows as $z^{2}$. This is in line with a volume geometry at extremely low redshift. In the righthand panel, one can see that the fraction of LSB and HSB galaxies in each bin is almost identical, although only bulgeless galaxies were selected there. These two figures suggest that the selection of bulgeless galaxies does not introduce any significant bias into the distribution of LSB and HSB galaxies. Figure 3 shows the relation between scale length and surface brightness for sample $S_{1}$. It classifies the sample into LSB galaxies and HSB galaxies. Most galaxies with surface brightness greater than 24 mag $\operatorname{arcsec}^{-2}$ are not included in this sample. This is probably due to the effective isophotal limit of the SDSS. Very diffuse galaxies below the detection limit cannot be identified. In Fig. 3, we also show a line corresponding to the luminosity cut for a mean profile.

\section{The environments of LSB and HSB galaxies}

The local density parameter $\Sigma_{5}$ (Rosenbaum \& Bomans 2004; Silverman et al. 2008; Padilla et al. 2010; Galaz et al. 2011) is used to estimate the density of the local environment. To obtain this parameter, we need to calculate the projected distance from an individual sample galaxy to the fifth nearest neighbor galaxy. During this step, the galaxies in the volume-limited main-galaxy 

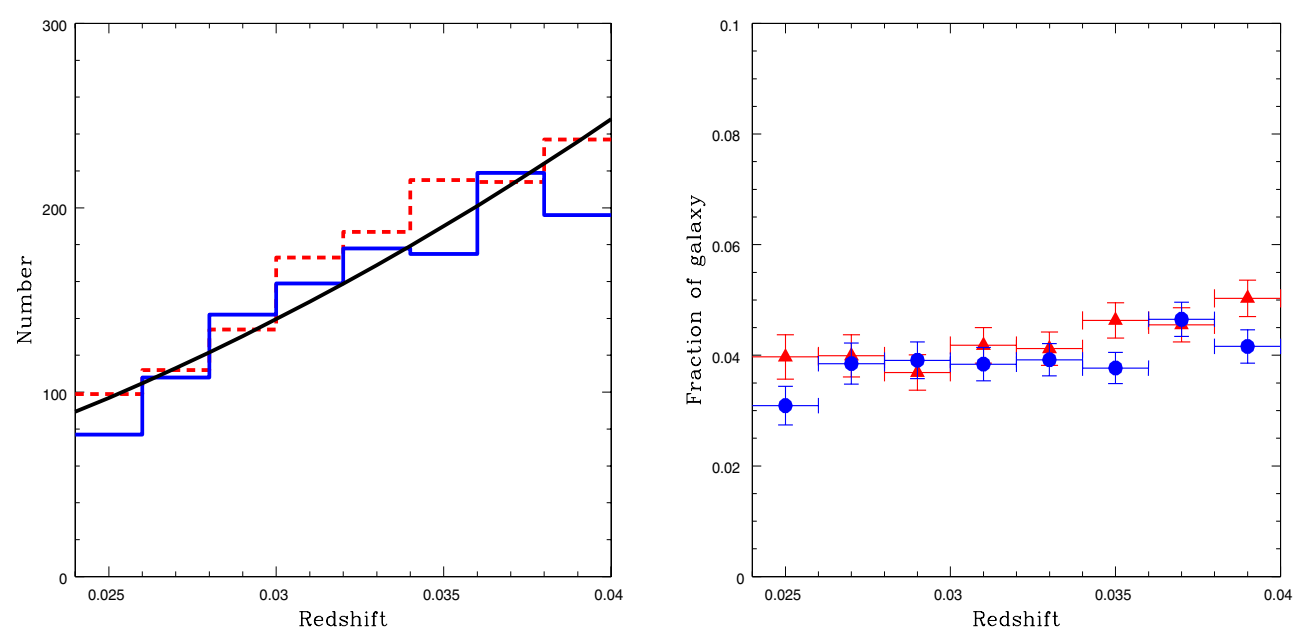

Fig. 2. Histogram distribution of redshifts for LSB (blue, sample $S_{\mathrm{LSB}}$ ) and HSB (red, sample $S_{\mathrm{HSB}}$ ) galaxies and their portion of the whole sample as a function of redshift. The black solid line denotes the expected number histogram of a complete volume-limited sample.

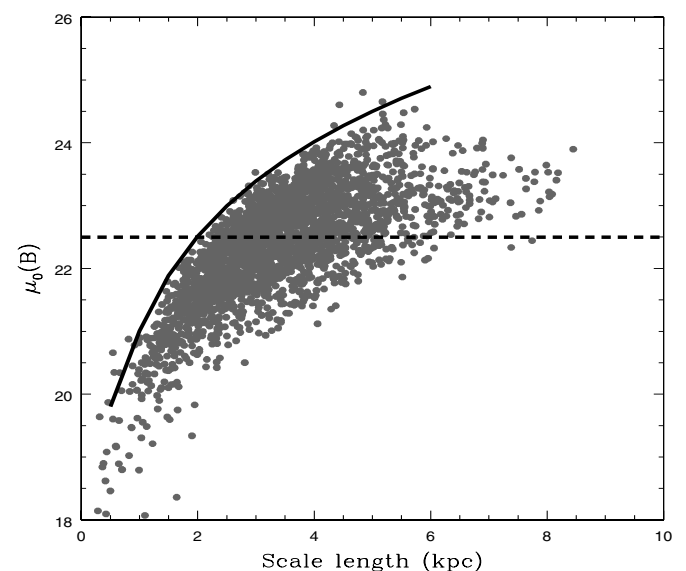

Fig. 3. Relation between scale length and surface brightness for sample $S_{1}$. The dashed line classifies the sample into LSB galaxies and HSB galaxies, and the solid line corresponds to the luminosity cut.

sample of SDSS DR7 (sample $S_{0}$ ), of which the $K$-corrected absolute magnitude are brighter than -18.8 (i.e. $M_{r} \leq-18.8$ ), are used as the tracers to count the $N$ th nearest neighbor. The selection of tracers is consistent with those of LSB and HSB sample galaxies in terms of limiting the absolute magnitude, so they will help to eliminate the luminosity bias. Here we would like to note that, for the tracers, we do not constrain the fracDeV and $b / a$. This means that not only the disk galaxies but also the galaxies with a bulge or elliptical galaxies are also included in this tracer sample. The information about the environment of one given galaxy should include all types of neighboring galaxies, because all of them will influence the formation and evolution of the target galaxies.

After the tracers are constructed, we are able to calculate the projected distance to the $N$ th nearest neighbor galaxies and then compute the local density parameter $\Sigma_{5}$. We define the $N$ th nearest neighbor within a velocity shell of $\pm 500 \mathrm{~km} \mathrm{~s}^{-1}$ and count the nearest neighbor from the first one to the fifth one. Then we get the projected distance of the fifth nearest neighbor $d_{5}$ in the unit of Mpc and define the local density by

$\Sigma_{5}=5 /\left(\pi d_{5}^{2}\right)$

in units of $\mathrm{Mpc}^{-2}$.
Whether the local density parameter $\Sigma_{5}$ can represent realistic environments of LSB and HSB galaxies needs to be investigated. We cross-correlate our volume-limited sample with the volume-limited galaxy group and clusters catalogs provided by Tempel et al. (2014). They used a modified friends-of-friends method with a variable linking length (LL) to identify the realistic groups/clusters with SDSS data release 10. For each galaxy, all neighbors within the LL radius are regarded as belonging to the same system. Their Vol-lim-18.0 catalog, where the $r$-band absolute magnitude limit is -18.0 and the LL radius is $0.38 \mathrm{Mpc}$, is consistent with the selection of our samples. We use objID to make the cross-correlation between their Vol-lim-18.0 catalog and our samples, and the parameter $N_{\text {gal }}$ is recovered. By $N_{\text {gal }}$ we denote the richness (number of members) of the groups or clusters to which the individual galaxy belongs, and it is a useful parameter for reflecting the realistic environment where LSB galaxies are located. Figure 4 shows the relation between $\log \left(N_{\text {gal }}\right)$ and $\log \left(\Sigma_{5}\right)$ for LSB galaxies and HSB galaxies. It also shows the median of $\log \left(\Sigma_{5}\right)$ for each $\log \left(N_{\text {gal }}\right)$, and the error bars show the dispersions. We can see that $\log \left(\Sigma_{5}\right)$ increases when $\log \left(N_{\mathrm{gal}}\right)$ becomes large. This relation is very clear when $\log \left(\Sigma_{5}\right)<1$, suggesting that the local density parameter $\Sigma_{5}$ is consistent with realistic environment parameter $N_{\text {gal }}$ and that it can reflect the density of the environment in which LSB and HSB galaxies are located. For the region with $\log \left(\Sigma_{5}\right)>1$, $d_{5}$ becomes less than $0.4 \mathrm{Mpc}$, which is comparable to or less than the LL radius $(0.38 \mathrm{Mpc})$, and the relation between $\log \left(\Sigma_{5}\right)$ and $\log \left(N_{\mathrm{gal}}\right)$ becomes less obvious.

In Fig. 5, we show the histogram distribution of $\log \left(\Sigma_{5}\right)$ for LSB galaxies and HSB galaxies. We can see that the distributions of $\log \left(\Sigma_{5}\right)$ are nearly the same, suggesting that the surface brightness of bulgeless galaxies does not depend on the environment.

\section{The stellar populations}

In this section, we study the stellar populations of LSB galaxies and see which factors, environment or dynamics, drive their evolution.

\subsection{Spectral synthesis analysis}

To investigate the evolution of LSB galaxies, we analyzed their stellar populations by fitting the full optical spectra using 

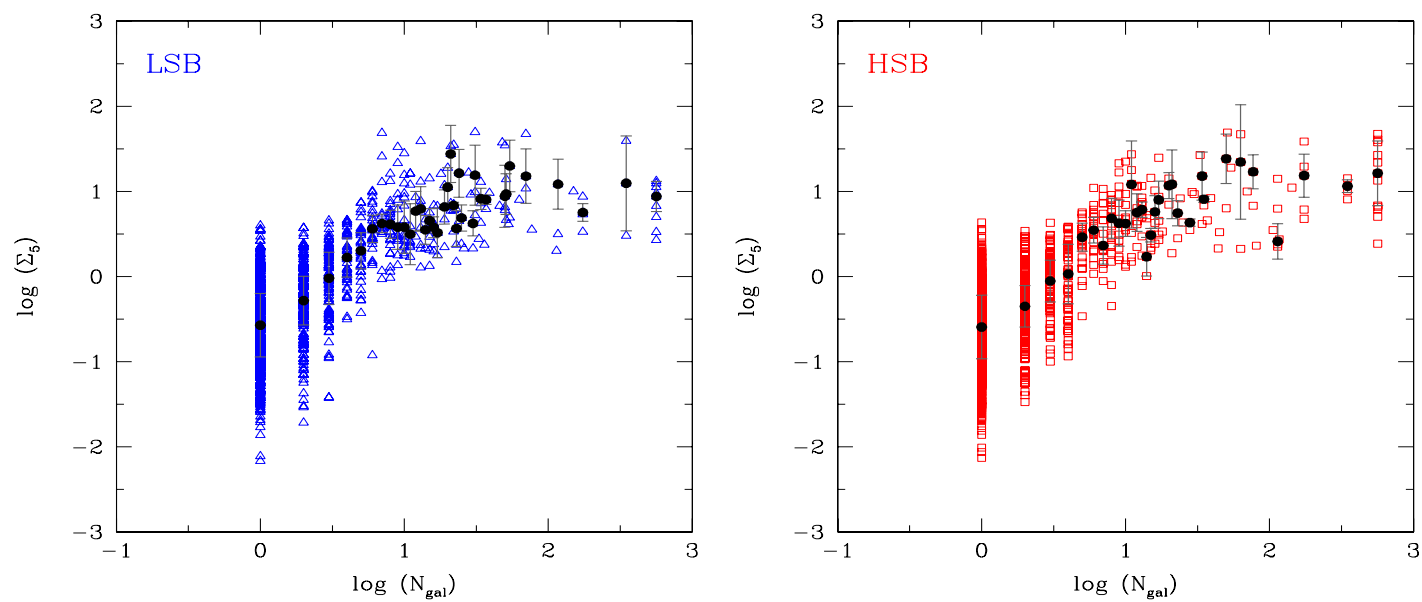

Fig. 4. Relation between $\log \left(N_{\text {gal }}\right)$ and $\log \left(\Sigma_{5}\right)$ for LSB galaxies (left panel, blue triangles) and HSB galaxies (right panel, red squares). The black dots give the median of $\log \left(\Sigma_{5}\right)$ for each $\log \left(N_{\mathrm{gal}}\right)$, and the error bars show the dispersions.

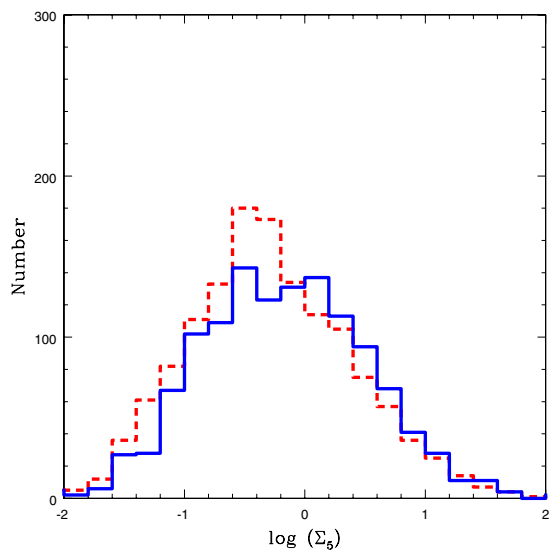

Fig. 5. Histogram distributions of $\log \left(\Sigma_{5}\right)$ for LSB galaxies (blue solid line) and HSB galaxies (red dashed line).

spectral synthesis. Spectral synthesis provides an efficient way to retrieve information about stellar populations of galaxies from observed spectra, which is very important for understanding the formation and evolution of galaxies. The information on both the age and metallicity distributions of stars contained in galaxy spectra can reflect the star formation and chemical histories of LSB galaxies. We analyzed the stellar populations of LSB galaxies to see which factor, environment or dynamics, affects their evolution.

We fit the absorption lines and continua of the spectrum of every individual galaxy using the software STARLIGHT (Cid Fernandes et al. 2005, 2007). This software fits an observed spectrum $O_{\lambda}$ with a model $M_{\lambda}$ that adds up $N_{*}$ simple stellar populations (SSPs) with different ages and metallicities taken from Bruzual \& Charlot (2003, BC03). In our analysis, we took 24 SSPs, including 12 different ages from 40 Myr to $13 \mathrm{Gyr}$ (40, 280, $900 \mathrm{Myr}$ and 1.27, 1.43, 2.5, 4.25, 5, 6.25, 7.5, 10, $13 \mathrm{Gyr})$ and two metallicities $\left(0.2\right.$ and $\left.1.0 Z_{\odot}\right)$, the stellar evolutionary tracks of Padova 1994 (Alongi et al. 1993; Girardi et al. 1996), the initial mass function (IMF) from Chabrier (2003), and the extinction law of Cardelli et al. (1989) with $R_{V}=3.1$. The Galactic extinctions were corrected by the reddening map of Schlegel et al. (1998) and the spectra shifted to the rest frame. The range of the spectra is from 3700 to $8000 \AA$ with a step of $1 \AA$ and normalized to the median flux in the 4010 to $4060 \AA$ region. During spectral synthesis fitting, we excluded the emission lines and four windows: (1) 5870-5905 $\AA$, to avoid the $N a D \lambda \lambda 5890,5896$ doublet, which is from the interstellar medium; (2) 6845-6945 $\AA$ and 7550-7725 $\AA$, which are the strong absorption bands from the Earth's atmosphere and were flagged by BC03 as problems in the STELIB library (Le Borgne et al. 2003); (3) 7165-7210 , which shows a systematic broad residual in emission as mentioned by Mateus et al. (2006; see also Chen et al. 2009, 2010).

As discussed in Cid Fernandes et al. (2005), the individual components of stellar populations fluctuate, but the combined stellar populations are robust enough. We need to combine the individual stellar population into a young population $(<1 \mathrm{Gyr})$, intermediate-age population $(1 \sim 5 \mathrm{Gyr})$, and old population ( $\geq 5 \mathrm{Gyr}$ ). When doing the spectral synthesis, we fit every spectrum 30 times with different random seeds to get a robust result and to estimate the associated uncertainty. For every individual galaxy, we take the median value of these 30 fitting results as a robust result and the standard deviation as the uncertainty. The typical value of the relative uncertainty of mass fraction in our sample is $13 \%, 21 \%$, and $30 \%$ for young, intermediate-age, and old stellar populations, respectively.

\subsection{Stellar population of LSB galaxies in different environments}

Figure 6 shows the histogram distributions of young, intermediate-age, and old stellar populations for LSB galaxies located in $\operatorname{low}\left(\log \left(\Sigma_{5}\right)<0.5\right.$ and $<-0.4$, i.e., $d_{5}>0.7$ and $2 \mathrm{Mpc}$, respectively) and high $\left(\log \left(\Sigma_{5}\right)>0.5\right)$ density region. The red solid line denotes LSB galaxies in a high density region and the blue solid line denotes LSB galaxies in a low density region while the blue dotted line delineates isolated LSB galaxies. Table 2 shows the median values of different stellar populations. We can see that, for young, intermediate-age, and old stellar populations, the distributions and the median value are nearly the same for LSB galaxies in low- and highdensity regions. On the other hand, Fig. 7 shows the histogram distributions of metal-poor stellar populations for LSB galaxies in low- and high-density regions. We cannot see obvious differences in metallicities for LSB galaxies in these three different environments. These results suggest that the stellar populations 

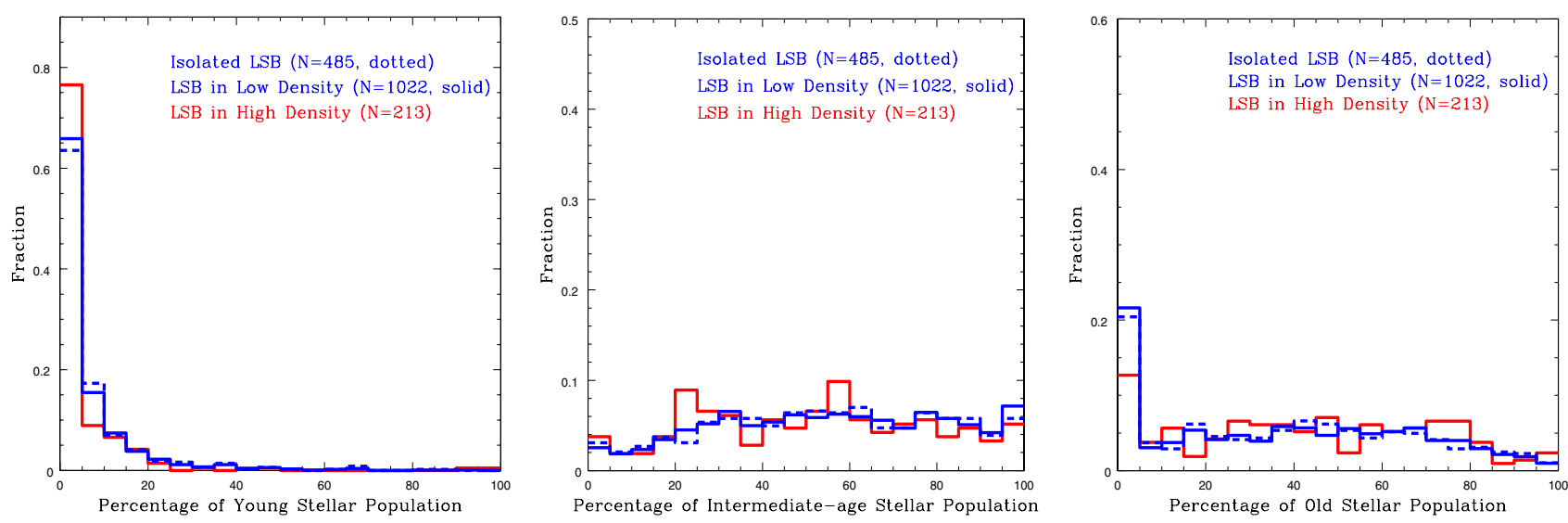

Fig. 6. Histogram distributions of young, intermediate-age, and old stellar populations for LSB galaxies located in a low-density region $\left(\log \left(\Sigma_{5}\right)<\right.$ $0.5)$ and a high-density region $\left(\log \left(\Sigma_{5}\right)>0.5\right)$. Isolated LSB galaxies are defined with $\log \left(\Sigma_{5}\right)<-0.4$.

Table 2. Median value of different stellar populations.

\begin{tabular}{cccc|c}
\hline \hline & \multicolumn{3}{c|}{ Age } & Metallicity \\
& young & intermediate & old & poor \\
\hline Isolated LSB & $3.3 \%$ & $55.5 \%$ & $38.3 \%$ & $74.9 \%$ \\
LSB in low density & $3.2 \%$ & $55.9 \%$ & $37.5 \%$ & $73.9 \%$ \\
LSB in high density & $2.2 \%$ & $53.6 \%$ & $42.2 \%$ & $68.2 \%$ \\
\hline Irregular LSB & $12.9 \%$ & $60.1 \%$ & $11.0 \%$ & $88.6 \%$ \\
Regular LSB & $2.7 \%$ & $55.1 \%$ & $40.5 \%$ & $71.0 \%$ \\
\hline
\end{tabular}

of LSB galaxies are similar when they are located in different environments. Environment is not found to play an important role in the evolution of LSB galaxies.

Whether these results are biased by the definition of low and high density needs to be checked. With two different definitions, we check the histogram distribution of stellar populations for LSB galaxies located in $\operatorname{low}\left(\log \left(\Sigma_{5}\right)<0, \log \left(\Sigma_{5}\right)<0.8\right)$ and high $\left(\log \left(\Sigma_{5}\right)>0, \log \left(\Sigma_{5}\right)>0.8\right)$ density regions. We find that there is still no obvious difference between the stellar populations of LSB galaxies in different environments, even with different definitions of low and high density. This suggests that the above results are not biased by the definition of low or high density and the similarities of stellar populations are real for LSB galaxies in different environments.

\subsection{Stellar population of LSB galaxies with different morphologies}

LSB galaxies exhibit a wide range of morphologies from dwarf irregular to luminous disk (McGaugh et al. 1995b). Like LSB galaxies in different environments, it is important to investigate whether the stellar populations of LSB galaxies with different morphologies are similar or not. To make this clear, we would like to compare the stellar populations of LSB galaxies that have different morphologies.

Morphological classification of LSB galaxies has been done by visual inspection of galaxy images from the SDSS. The whole LSB sample is classified into two groups: regular LSB galaxies and irregular LSB galaxies. Most LSB galaxies can be classified correctly, except some very small ones because of the spatial resolution. The classification has been done by XS and $\mathrm{FH}$ and some examples of irregular galaxies are shown at the end of the paper (Figs. 12 and 13). The major difficulty is to distinguish Irr

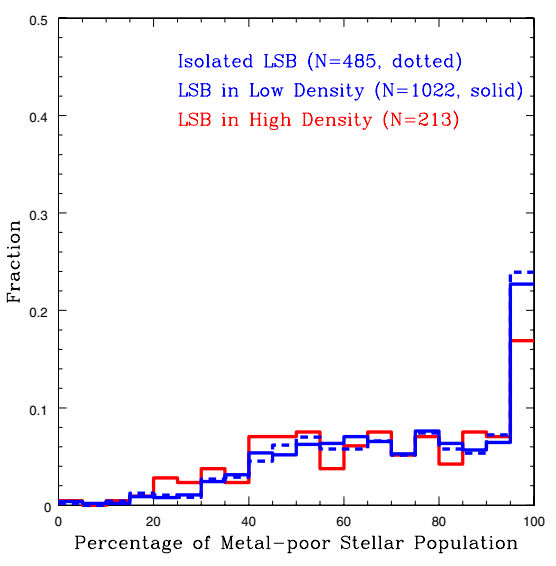

Fig. 7. Histogram distributions of metal-poor stellar populations for LSB galaxies located in a low-density region $\left(\log \left(\Sigma_{5}\right)<0.5\right)$ and a high-density region $\left(\log \left(\Sigma_{5}\right)>0.5\right)$. Isolated LSB galaxies are defined with $\log \left(\Sigma_{5}\right)<-0.4$.

from SBm (very late type galaxies), and this is certainly the main uncertainty in this visual classification.

Figure 8 shows the histogram distributions of young, intermediate-age, and old stellar populations for regular and irregular LSB galaxies. Table 2 shows median values of these distributions. The differences are significant. Irregular LSB galaxies have more young stellar populations and less old stellar populations than regular LSB galaxies, suggesting that they are younger and have more young stars than regular LSB galaxies. For the intermediate-age stellar populations, the differences are not obvious. Figure 9 shows the histogram distributions of metalpoor stellar populations for regular and irregular LSB galaxies. Regular LSB galaxies have less metal-poor stellar populations than irregular LSB galaxies. These results suggest that irregular LSB galaxies formed more stars than regular LSB galaxies at recent epochs. The increase in metallicity in regular LSB galaxies may go faster than in irregular LSB galaxies.

\section{Discussion}

\subsection{Differences in environments between LSB and HSB galaxies}

In the above analysis, we found that the surface brightness of bulgeless galaxies does not depend on the environment. We 
X. Shao et al.: Stellar populations of bulgeless LSB galaxies
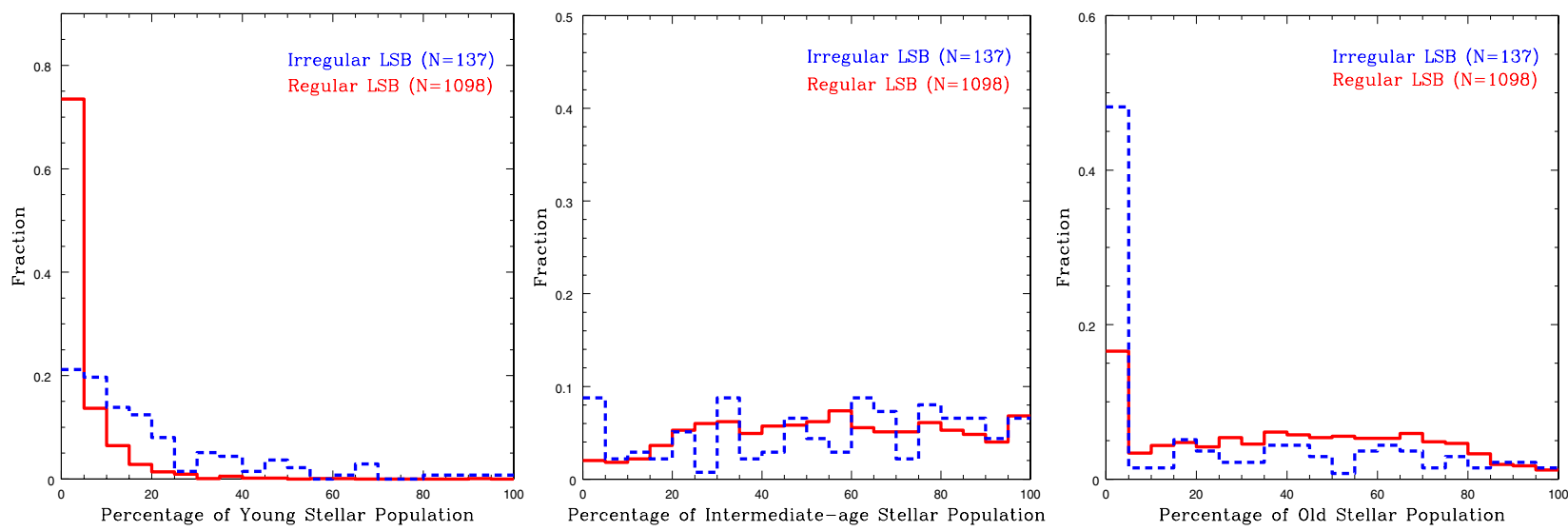

Fig. 8. Histogram distributions of young, intermediate-age, and old stellar populations for regular LSB galaxies and irregular LSB galaxies. Regular LSB galaxies have almost no young stellar component compared to morphologically peculiar LSB galaxies that cover large sections of young stellar populations.

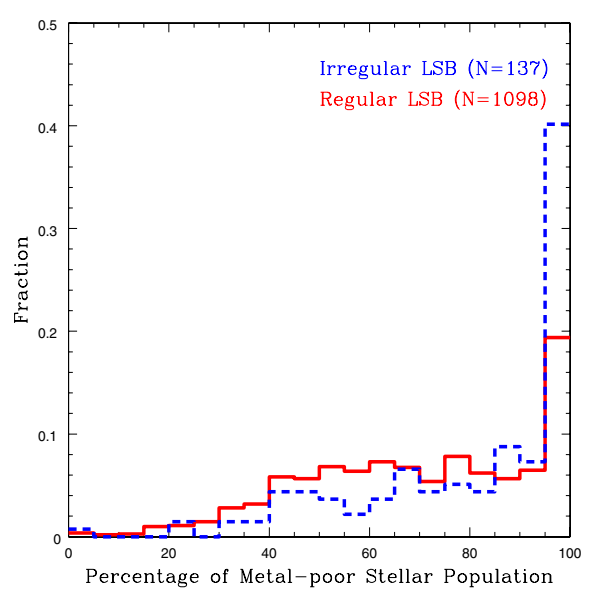

Fig. 9. Histogram distributions of metal-poor stellar populations for regular LSB galaxies and irregular LSB galaxies.

notice that this appears to be different from previous studies. Bothun et al. (1993) and Mo et al. (1994) found that LSB galaxies are located in more isolated environments than HSB galaxies. The differences are probably due to the different selection criteria. They selected not only bulgeless galaxies but also galaxies with bulges, but we only selected bulgeless galaxies. However, only selecting bulgeless LSB and HSB galaxies, which are latetype galaxies, should not bring a significant bias when comparing their environments. Indeed, massive galaxies are located in higher density regions and have higher bulge-to-total ratio $(\mathrm{B} / \mathrm{T})$ than low-mass galaxies (Hammer et al. 2005). This suggests that galaxies with high $\mathrm{B} / \mathrm{T}$ may be located in high-density regions. Considering the large number of HSB galaxies with bulges, which may make them have higher average density than LSB galaxies, previous works may have bias when comparing their environment by selecting galaxies with bulges.

\subsection{Aperture effect in spectral synthesis}

As is well known, SDSS is a fiber-based survey. The spectra of objects are taken by fibers with diameters of 3 arcsec. When extended sources are analyzed, such as nearby galaxies, there are aperture effects. Tremonti et al. (2004) and Kewley et al. (2005) discuss the aperture effects of SDSS spectroscopy in detail. To check how much light is covered by SDSS observation, one simple and accurate way is to compare the fiber magnitude and Petrosian magnitude of our samples. The fiber magnitude is a measurement of the light falling into the fiber, and the Petrosian magnitude is a good estimate of the total light. Thus, we adopt the formula below (Hopkins et al. 2003) to estimate how much light is covered by fiber observations:

$f_{\text {light }}=10^{\left(-0.4\left(m_{\text {fiber }}-m_{\text {petro }}\right)_{r}\right)}$.

The typical value of $f_{\text {light }}$ is $\sim 0.08$, indicating that the light of the spectra comes from the inner regions of galaxies. Generally, there are age and/or metallicity gradients in LSB galaxies (de Jong 1996; Bell et al. 2000). Inner regions have old and metal-rich stellar populations, while young and metal-poor stellar populations dominate outer regions, so our estimations of the stellar populations represent the properties of the central regions. The whole disk of LSB galaxies could be younger and more metal-poor than are central regions. On the other hand, our samples consist of bulgeless galaxies, and the spectra sample only have the central part of the disks, so our results represent the properties of central disks well without contaminations from bulge light.

To check whether the above results are biased by different aperture effects, we show the histogram distributions of $f_{\text {light }}$ for different LSB galaxies in Fig. 10. We can see that, for LSB galaxies in different environments or with different morphologies, the distributions are nearly the same, suggesting that our results are not biased by different aperture effects and are robust enough.

\subsection{The origin of star formation in irregular LSB galaxies}

Table 2 shows the median values of different stellar populations for irregular LSB galaxies and regular LSB galaxies. Irregular LSB galaxies have more young and metal-poor stellar populations in the center than regular LSB galaxies. Because of the degeneracy between age and metallicity, it is difficult to analyze the star formation histories in detail using these results. But for a young stellar population with poor metallicity, there is no degeneracy. Generally, the central region of disk galaxies has old and metal-rich stellar populations, and the outer region has young and metal-poor stellar populations (de Jong 1996; Bell et al. 2000). It is interesting that the irregular LSB galaxies have such 

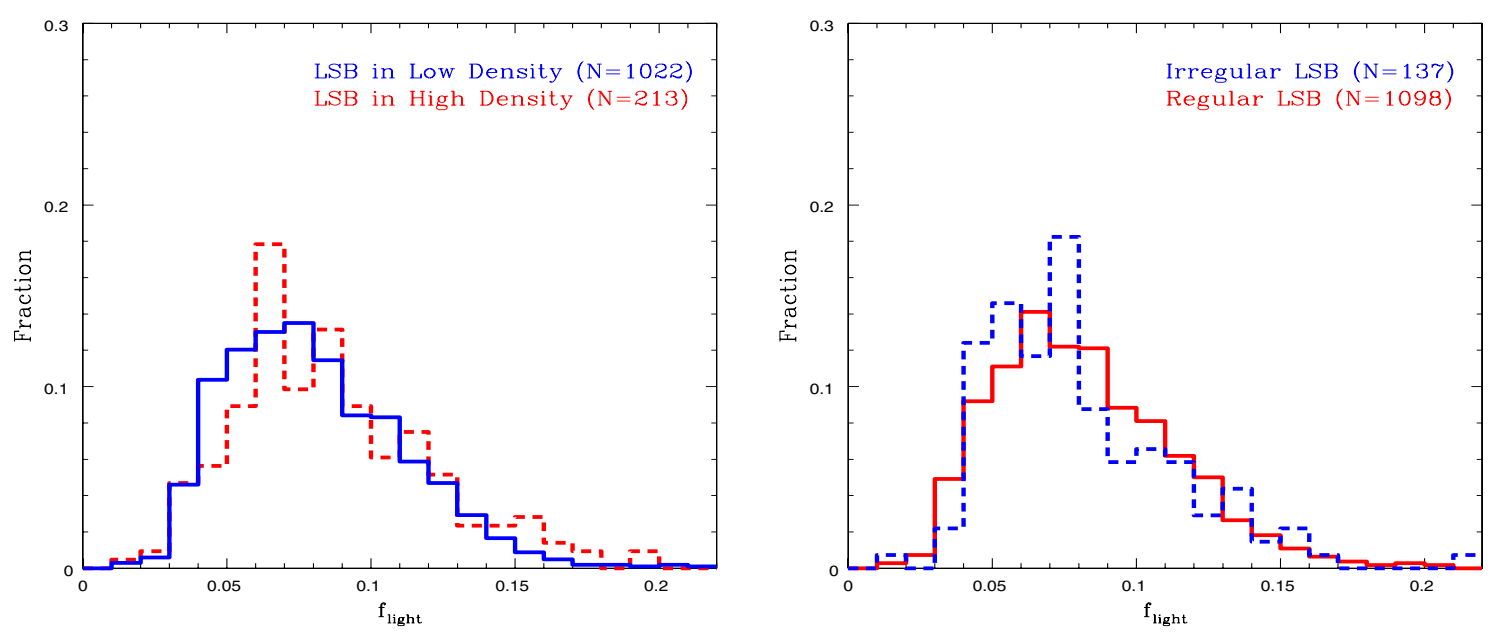

Fig. 10. Histogram distributions of light fraction for different LSB galaxies.

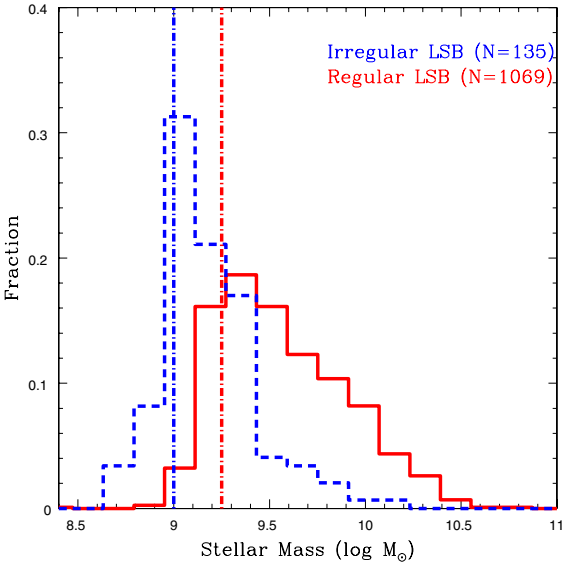

Fig. 11. Histogram distributions of stellar mass for regular LSB galaxies and irregular LSB galaxies. The red and blue dot-dashed lines denote the mass-completeness limits of regular and irregular LSB galaxies, respectively.

a high percentage of young stars $(<1 \mathrm{Gyr})$ in their central regions. What kind of mechanisms can make young stars appear in the center of irregular LSB galaxies?

The detection of young stars suggests that the central regions of irregular LSB galaxies may experience recent star formation episodes, which result in the so-called clumps. What drives the formation of clumps has been widely studied. After analyzing the kinematics of clumpy galaxies at $z \sim 0.6$, Puech (2010) suggests that cold flows are expected to feed $z>1$ clumpy galaxies, while mergers are probably the dominant driver of the formation of clumpy galaxies at $z<1$. For our sample with redshifts ranging from 0.024 to 0.04 , mergers may be one of the reasons for the appearance of irregularities and central star formations.

Figure 11 shows the histogram distributions of stellar masses for regular and irregular LSB galaxies. The stellar masses are recovered from MPA-JHU ${ }^{2}$ and estimated using fits to the photometry. Irregular, star-forming galaxies have lower stellar mass than others. This is probably due to the different mass-to-light ratios $(\mathrm{M} / \mathrm{L})$ of these two subsamples. Such variations could be presented when we found the differences in the fraction of young stars. To estimate this incompleteness, we determined

\footnotetext{
2 http://www .mpa-garching.mpg.de/SDSS/DR7/
}

the mean stellar mass of the galaxies that are located very near the luminosity cut (between -19 and -18.8) and considered that as a mass-completeness limit. Figure 11 shows the masscompleteness limits of irregular and regular LSB galaxies, which are 9.0 and 9.25, respectively. The LSB galaxies with stellar mass higher than these limits are considered to be complete in stellar mass. We focus in the following on irregular LSB galaxies with $\log M_{\odot}>9.5$ (to compare to the regular ones), as well as on the irregular ones with $\log M_{\odot}<9.0$.

For massive LSB galaxies ( $\left.\log M_{\odot}>9.5\right)$, it is normally difficult for the gas to fall into the center and form stars. One possible mechanism for forming young stars in the central region is through a gas-rich merger that could transport gas from the outskirts of the galaxy into the center (Hammer et al. 2009; Rodrigues et al. 2008, 2012). For more than half of the irregular LSB galaxies in Fig. 12, we see a merger occurring within them when checking their images. For the irregular LSB galaxies with $\log M_{\odot}>9.5$ in our sample, we also find seven with bars appearing in the center, a phenomenon that can be enhanced by merging in peculiar galaxies. The presence of a bar is believed to be an efficient way to drive gas toward the central region of a galaxy (Norman et al. 1996), which may trigger strong star formation (Haan et al. 2009).

McConnachie (2012) reviewed nearly 100 nearby dwarf galaxies and found that most of them have stellar mass less than $10^{9} M_{\odot}$. When checking the images of irregular LSB galaxies with $\log M_{\odot}<9.0$ in Fig. 13, we find that most of them are dwarf irregulars (dIrrs). Several studies have revealed that recent star formation in dIrrs takes place at their center (Wilcots \& Miller 1998; Miller et al. 2001; Garcia et al. 2009; Lianou \& Cole 2013; Fouquet et al. 2015). These star formation episodes might be triggered by the collapse of high-density gas. Indeed, Momany et al. (2005) found that young stars are located near regions with high HI column density in the Sagittarius dwarf irregular galaxy. There is still the possibility that the low-mass irregular LSB galaxies are tidal dwarf galaxies (TDGs), which can form young stars in their center (Hammer et al. 2013; Fouquet et al. 2015). The situation is more complicated for irregular LSB galaxies with $9.0<\log M_{\odot}<9.5$. Kinematics need to be done in detail to see why they have a large number of young stars in their central region.

From this quite crude analysis in the absence of support from kinematics, it seems that LSB galaxies share most of their properties with HSB galaxies. Mostly depending on their masses, the 

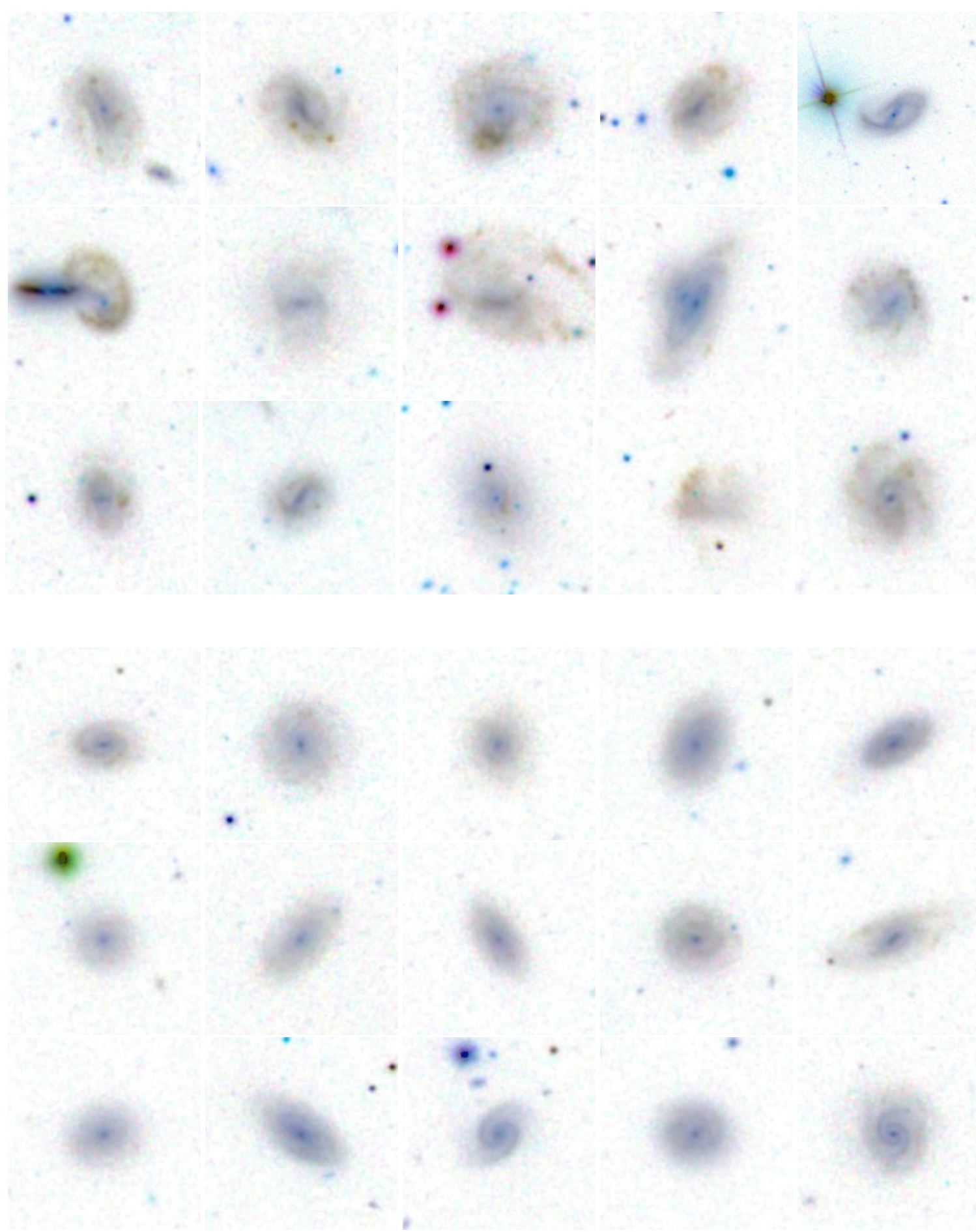

Fig. 12. Images of high-mass irregular LSB galaxies $\left(\log M_{\odot}>9.5\right.$, top panel) and high-mass regular LSB galaxies $\left(\log M_{\odot}>9.5\right.$, bottom panel). From top left to bottom right in the top panel, the 3rd, 5th, 6th, 8th, 9th, 10th, 12th, and 15th show that either multiple components, double nuclei, or tidal features are all proof of merging.

star formation history of the most massive ones are affected by past mergers, while the least massive ones are gradually more affected by stellar phenomena (e.g., outflows) and minor mergers.

\section{Conclusion}

The environments in which bulgeless LSB galaxies and bulgeless HSB galaxies are located were compared, and the stellar populations of bulgeless LSB galaxies were investigated in this work. We selected a large sample of 1235 bulgeless low-inclination LSB galaxies in a volume-limited sample with redshifts ranging from 0.024 to 0.04 and $M_{r} \leq-18.8$. The local density parameter $\Sigma_{5}$ is calculated to trace their environment. This parameter gives a hint of how far the nearest neighbors are away from the galaxy, but it does not provide any insight into what interactive histories the galaxies might have had. For bulgeless galaxies, we find that their surface brightness does not depend on the environment. Compared with previous studies, only selecting bulgeless galaxies avoids significant bias when comparing their environments. 


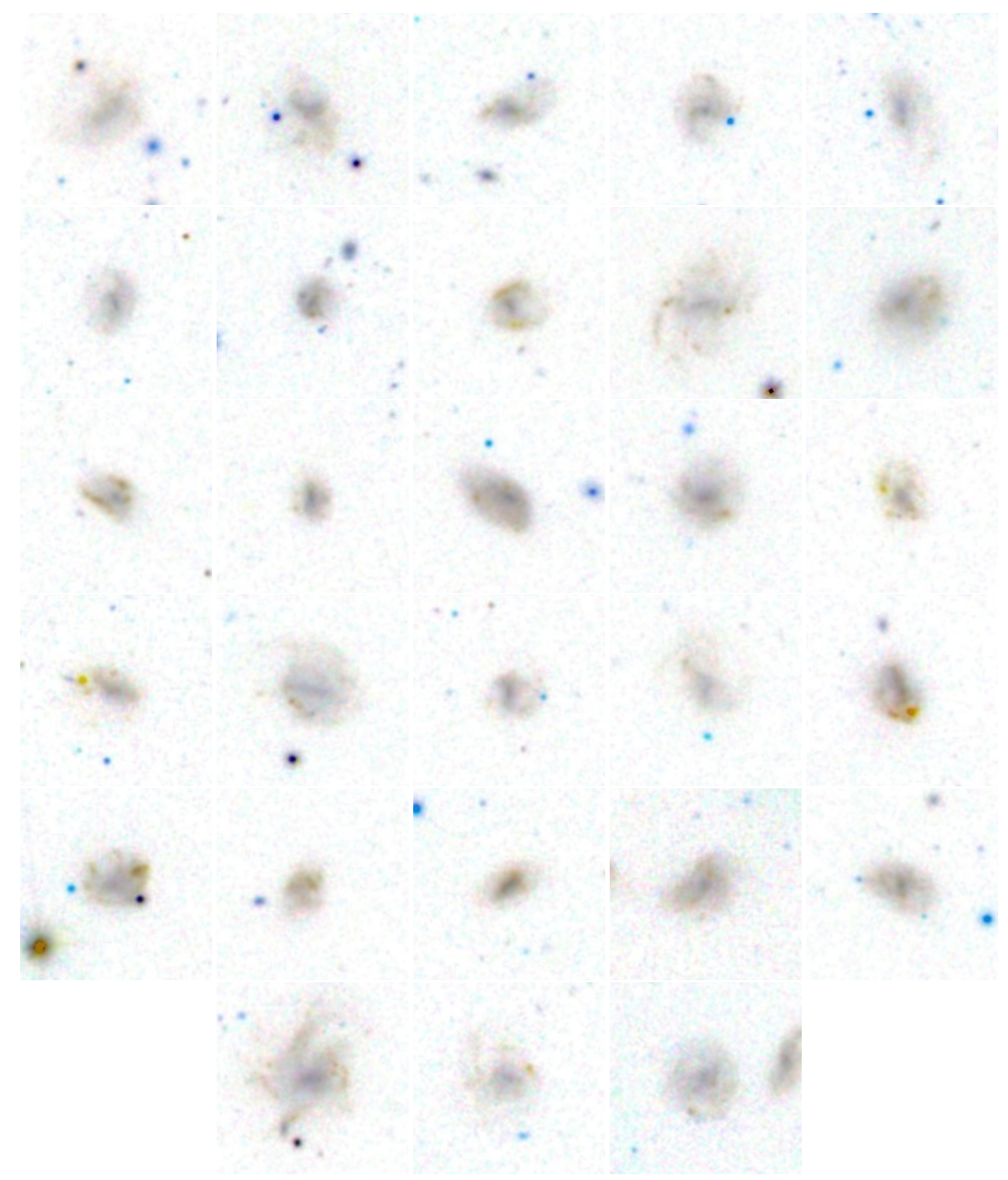

Fig. 13. Images of low-mass irregular LSB galaxies $\left(\log M_{\odot}<9.0\right)$.

The stellar populations of bulgeless LSB galaxies in lowdensity regions are similar to those of bulgeless LSB galaxies in high-density regions, suggesting that the environment may play a less important role in the evolution of bulgeless LSB galaxies. On the other hand, bulgeless LSB galaxies with different morphologies have significant differences in the stellar populations. Different dynamic situations make irregular LSB galaxies have more young stars in the center than regular LSB galaxies. These results suggest that the dynamics and mergers for at least the more massive ones may play a dominant role in the evolution of LSB galaxies rather than environment.

Acknowledgements. We thank James Wicker for improving our English expression in the text from the native language. We appreciate Hector Flores, Guohu Zhong, Minnie Lam, and Ming Yang for helpful discussions about this paper. This study was supported by the Natural Science Foundation of China under grants Nos. 11273026 and 10933001. Funding for the SDSS and SDSS-II was provided by the Alfred P. Sloan Foundation, the Participating Institutions, the National Science Foundation, the US Department of Energy, the National Aeronautics and Space Administration, the Japanese Monbukagakusho, the Max Planck Society, and the Higher Education Funding Council for England. The SDSS Web Site is http://www.sdss.org/. The SDSS is managed by the Astrophysical Research Consortium for the Participating Institutions. The Participating Institutions are the American Museum of Natural History, Astrophysical Institute Potsdam, University of Basel, University of Cambridge, Case Western Reserve University, University of Chicago, Drexel University, Fermilab, the Institute for Advanced Study, the Japan Participation Group, Johns Hopkins University, the Joint Institute for Nuclear Astrophysics, the Kavli Institute for Particle Astrophysics and Cosmology, the Korean Scientist Group, the Chinese Academy of Sciences (LAMOST), Los Alamos National Laboratory, the Max-Planck-Institute for Astronomy (MPIA), the Max-Planck-Institute for Astrophysics (MPA), New Mexico State University, 
Ohio State University, University of Pittsburgh, University of Portsmouth, Princeton University, the United States Naval Observatory, and the University of Washington.

\section{References}

Abazajian, K. N., Adelman-McCarthy, J. K., Agüeros, M. A., et al. 2009, ApJS, 182,543

Alongi, M., Bertelli, G., Bressan, A., et al. 1993, A\&AS, 97, 851

Bell, E. F., Barnaby, D., Bower, R. G., et al. 2000, MNRAS, 312, 470

Bothun, G. D., Schombert, J. M., Impey, C. D., Sprayberry, D., \& McGaugh, S. S. 1993, AJ, 106, 530

Bruzual, G., \& Charlot, S. 2003, MNRAS, 344, 1000

Caldwell, N., \& Bothun, G. D. 1987, AJ, 94, 1126

Cardelli, J. A., Clayton, G. C., \& Mathis, J. S. 1989, ApJ, 345, 245

Chabrier, G. 2003, PASP, 115, 763

Cid Fernandes, R., González Delgado, R. M., Storchi-Bergmann, T., Martins, L. P., \& Schmitt, H. 2005, MNRAS, 356, 270

Cid Fernandes, R., Asari, N. V., Sodré, L., et al. 2007, MNRAS, 375, L16

Chen, X. Y., Liang, Y. C., Hammer, F., Zhao, Y. H., \& Zhong, G. H. 2009, A\&A, 495, 457

Chen, X. Y., Liang, Y. C., Hammer, F., et al. 2010, A\&A, 515, A101

de Blok, W. J. G., van der Hulst, J. M., \& Bothun, G. D. 1995, MNRAS, 274,235

de Jong, R. S. 1996, A\&A, 313, 377

Delgado-Serrano, R., Hammer, F., Yang, Y. B., et al. 2010, A\&A, 509, A78

Disney, M. J. 1976, Nature, 263, 573

Haan, S., Schinnerer, E., Emsellem, E., et al. 2009, ApJ, 692, 1623

Fouquet, S., et al. 2015, MNRAS, submited

Galaz, G., Herrera-Camus, R., Garcia-Lambas, D., \& Padilla, N. 2011, ApJ, 728,74

Garcia, M., Herrero, A., Vicente, B., et al. 2009, A\&A, 502, 1015

Girardi, L., Bressan, A., Chiosi, C., Bertelli, G., \& Nasi, E. 1996, A\&AS, 117,113

Governato, F., Brook, C., Mayer, L., et al. 2010, Nature, 463, 203

Hammer, F., Flores, H., Elbaz, D., et al. 2005, A\&A, 430, 115

Hammer, F., Flores, H., Puech, M., et al. 2009, A\&A, 507, 1313

Hammer, F., Yang, Y., Flores, H., \& Puech, M. 2012 [arXiv: 1211. 1978]

Hammer, F., Yang, Y., Fouquet, S., et al. 2013, MNRAS, 431, 3543

Hopkins, A. M., Miller, C. J., Nichol, R. C., et al. 2003, ApJ, 599, 971

Impey, C., \& Bothun, G. 1997, ARA\&A, 35, 267

Keselman, J. A., \& Nusser, A. 2012, MNRAS, 424, 1232

Kewley, L. J., Jansen, R. A., \& Geller, M. J. 2005, PASP, 117, 227

Kormendy, J., Drory, N., Bender, R., \& Cornell, M. E. 2010, ApJ, 723, 54
Le Borgne, J.-F., Bruzual, G., Pelló, R., et al. 2003, A\&A, 402, 433

Liang, Y. C., Zhong, G. H., Hammer, F., et al. 2010, MNRAS, 409, 213

Lianou, S., \& Cole, A. A. 2013, A\&A, 549, A47

Mateus, A., Sodré, L., Cid Fernandes, R., et al. 2006, MNRAS, 370, 721

McConnachie, A. W. 2012, AJ, 144, 4

McGaugh, S. S., Bothun, G. D., \& Schombert, J. M. 1995a, AJ, 110, 573

McGaugh, S. S., Schombert, J. M., \& Bothun, G. D. 1995b, AJ, 109, 2019

Miller, B. W., Dolphin, A. E., Lee, M. G., Kim, S. C., \& Hodge, P. 2001, ApJ, 562,713

Mo, H. J., McGaugh, S. S., \& Bothun, G. D. 1994, MNRAS, 267, 129

Momany, Y., Held, E. V., Saviane, I., et al. 2005, A\&A, 439, 111

Morshidi-Esslinger, Z., Davies, J. I., \& Smith, R. M. 1999, MNRAS, 304, 297

Neichel, B., Hammer, F., Puech, M., et al. 2008, A\&A, 484, 159

Norman, C. A., Sellwood, J. A., \& Hasan, H. 1996, ApJ, 462, 114

O’Neil, K., \& Bothun, G. 2000, ApJ, 529, 811

O’Neil, K., Bothun, G. D., \& Cornell, M. E. 1997, AJ, 113, 1212

Padilla, N., Lambas, D. G., \& González, R. 2010, MNRAS, 409, 936

Phillipps, S., Davies, J. I., \& Disney, M. J. 1990, MNRAS, 242, 235

Puech, M. 2010, MNRAS, 406, 535

Puech, M., Hammer, F., Hopkins, P. F., et al. 2012, ApJ, 753, 128

Rodrigues, M., Hammer, F., Flores, H., et al. 2008, A\&A, 492, 371

Rodrigues, M., Puech, M., Hammer, F., Rothberg, B., \& Flores, H. 2012, MNRAS, 421, 2888

Rosenbaum, S. D., \& Bomans, D. J. 2004, A\&A, 422, L5

Rosenbaum, S. D., Krusch, E., Bomans, D. J., \& Dettmar, R.-J. 2009, A\&A, 504,807

Sachdeva, S. 2013, MNRAS, 435, 1186

Schlegel, D. J., Finkbeiner, D. P., \& Davis, M. 1998, ApJ, 500, 525

Schombert, J. M., \& Bothun, G. D. 1988, AJ, 95, 1389

Schombert, J. M., Bothun, G. D., Schneider, S. E., \& McGaugh, S. S. 1992, AJ, 103,1107

Silverman, J. D., Mainieri, V., Lehmer, B. D., et al. 2008, ApJ, 675, 1025

Smith, J. A., Tucker, D. L., Kent, S., et al. 2002, AJ, 123, 2121

Stoughton, C., Lupton, R. H., Bernardi, M., et al. 2002, AJ, 123, 485

Strauss, M. A., Weinberg, D. H., Lupton, R. H., et al. 2002, AJ, 124, 1810

Tempel, E., Tamm, A., Gramann, M., et al. 2014, A\&A, 566, A1

Tremonti, C. A., Heckman, T. M., Kauffmann, G., et al. 2004, ApJ, 613, 898

Unterborn, C. T., \& Ryden, B. S. 2008, ApJ, 687, 976

Walcher, C. J., van der Marel, R. P., McLaughlin, D., et al. 2005, ApJ, 618,237

Wilcots, E. M., \& Miller, B. W. 1998, AJ, 116, 2363

York, D. G., Adelman, J., Anderson, J. E., Jr., et al. 2000, AJ, 120, 1579

Zaritsky, D., \& Lorrimer, S. J. 1993, 72. The Evolution of Galaxies and Their Environment, Proc. NASA. Ames Res. Center, 82

Zhong, G. H., Liang, Y. C., Liu, F. S., et al. 2008, MNRAS, 391, 986 